\title{
Some new evidence on human joint lubrication*
}

\author{
A. UNSWORTH, D. DOWSON, AND V. WRIGHT \\ From the Bioengineering Group for the Study of Human Joints, Leeds University
}

\begin{abstract}
Unsworth, A., Dowson, D., and Wright, V. (1975). Annals of the Rheumatic Diseases, 34, 277-285. Some new evidence on human joint lubrication. Theoretical consideration has been given to the use of pendulum machines which are used to examine the frictional properties of human joints by incorporating them as fulcra. As a result, a new type of pendulum machine has been built which incorporates the facility to apply sudden loads to the joint on starting the swinging motion, and also the ability to measure directly the frictional torque experienced by the joint.

The results obtained from natural hip joints indicate the presence of squeeze film lubrication under conditions of sudden loading of a joint. In addition, a self-generated fluid film process was observed at low loads while at higher loads boundary lubrication appeared to be important. These results have been used to describe the lubrication regimens occurring in a normal activity such as walking.

A single experiment carried out on a hip from a patient suffering from severe rheumatoid arthritis has also been reported and the frictional resistance was seen to be increased fifteenfold compared to a normal hip.
\end{abstract}

The exact mechanisms of lubrication present in human joints have been sought for many years and there is an unresolved difference of opinion as to whether fluid film or boundary lubrication is more important.

In support of fluid film lubrication, MacConaill (1932) published his anatomical studies of the knee joint, showing the applicability of geometry to hydrodynamic lubrication. Jones (1936) supported this view of hydrodynamic lubrication, having carried out tests in which an interphalangeal joint was made the fulcrum of a pendulum machine. The resulting motion was seen to have an exponential decay in amplitude which he attributed to fluid film lubrication.

McCutchen (1959) proposed a novel type of fluid film action termed 'weeping lubrication'. Here the synovial fluid-filled cartilage weeps under load to pressurize the fluid and force it from the cartilage interstices into the joint space to provide a lubricant to separate the surfaces of the joint. Dintenfass (1963), Tanner (1966), and Dowson (1966-67) all proposed elastohydrodynamic lubrication since the cartilage was thought to deform substantially under load, and Dowson calculated that this form of fluid film mechanism was the only one to predict practical film thickness dimensions.

Maroudas (1966-67) showed that synovial fluid gels form on the surface of cartilage when subjected to pressure, and Walker, Dowson, Longfield, and Wright (1968) proposed a modified form of squeeze film lubrication whereby the molecular structure of synovial fluid and the elasticity, porosity, and surface topography of articular cartilage contrived to prolong the life of effective lubricating films; a type they termed 'boosted lubrication'.

In contrast, Charnley $(1959,1960)$ predicted boundary lubrication and suggested that Jones (1936) had been confused by the effects of soft tissue surrounding the joint which resisted the motion of the pendulum machine. In his own pendulum experiments in which a cadaveric ankle joint was used as fulcrum, Charnley (1960) showed that the decay in amplitude was linear and therefore that boundary lubrication was operative. Little, Freeman, and Swanson (1969) supported Charnley's view and conducted their own pendulum experiments using cadaveric hip joints as fulcra. Again the decay in amplitude with time was seen to be linear and the authors predicted boundary lubrication. 
McCutchen (1966) showed that while weeping lubrication was important in joints, an obvious boundary lubricant was also present. He carried out various tests using synovial fluid as a lubricant for rubber against glass, and by careful design of the sliding surfaces he eliminated any fluid film action and showed that adsorption on the surfaces undergoing sliding was important. McCutchen concluded that the boundary lubrication was very important in the highly loaded articular surfaces while fluid film lubrication was important in soft tissue lubrication.

Linn and Radin (1968), Linn (1968, 1969), and Radin, Swann, and Weisser (1970) treated synovial fluid with hyaluronidase which broke the hyaluronic acid into smaller chain lengths and consequently reduced the viscosity. In spite of this, when tested the coefficient of friction remained low. However, when the protein fraction of the synovial fluid was digested by trypsin, the synovial fluid failed to lubricate cartilage as effectively as before even though the viscosity was unchanged. From this the authors concluded that the protein acted as a boundary lubricant and that this was the mechanism of human joint lubrication.

Clearly, then, there is disagreement between various workers, and an interesting observation by Barnett and Cobbold (1962) justifies another look at pendulum machines. They observed that Charnley (1960) was correct in stating that the soft tissue was responsible for the exponential nature of the decay in amplitude of a pendulum when an intact joint was used as the fulcrum. This was verified by selectively dissecting tissue until the joint surfaces alone remained. In this condition the amplitude-time relationship became linear. However, the most interesting observation in these experiments was that when a hydrostatic bearing was used as a fulcrum, the amplitude decay was also linear. In this case the authors knew that a fluid film existed between the two sliding surfaces of the fulcrum yet theoretically the amplitude suggested that no film existed. This is a very important aspect of pendulum tests and could invalidate certain observations and conclusions obtained from such tests.

The present communication re-examines the approach to studies of synovial joint lubrication based upon the pendulum type of testing machines and shows that such machines, when the data are carefully analysed, can give valuable information on the modes of lubrication present in natural and artificial joints.

\section{Study of the pendulum machine}

Pendulum machines in which human joints form the fulcra have been shown to be simple experimental machines to predict the modes of lubrication present within the joints. If the relationship between the angular displacement and time of swing of such a pendulum is known, then basic assumptions can be made about the nature of the resistive elements acting at the fulcrum.

If the fulcrum exhibits no frictional resistance at $\frac{}{6}$. all then the resulting motion of the pendulum is simple harmonic in nature. This means that the decay in amplitude will be zero and the pendulum will $\overline{0}$ carry on swinging indefinitely. If, however, a constant $\frac{}{0}$ frictional resistance is present in the fulcrum, such as $\frac{\bar{m}}{\vec{D}}$ that produced by classical boundary friction, then the decay in amplitude will become linear. In other words, the amplitude of swing of the pendulum reduces by a fixed amount each oscillation until the pendulum comes to rest.

A third possibility is to have a viscous resistance ${ }^{\omega}$ exhibited by the fulcrum. In this case the resistance is dependent on the velocity of swing of the pendulum which reduces as the amplitude reduces. Here the decay in amplitude can be shown to have an exponential form which theoretically never actually comes to $\searrow$ rest. In practice other factors influence this motion 0 causing it to stop after a definite time. To explain this mathematically consider the pendulum shown in Fig. 1. The mass $m$ of the pendulum can be considered to be concentrated at a radius $R$ from the $\frac{c}{\infty}$ fulcrum, while the angular displacement $\theta$ is measured from the vertical line shown. If we assume that $\theta$ at the fulcrum of such a pendulum a viscous and coulomb resistance exist, then the equation of motiom governing this situation becomes:

$$
m R^{2 d^{2} \theta}-f \frac{d \theta}{d t}-m R g \theta \pm k=0 \text { (for small angles } \theta \text { ), }
$$

where $f$ is the viscous damping coefficient, $k$ is $\frac{\mathbb{Q}}{\Rightarrow}$ constant coulomb frictional torque, and $g$ is gravitational acceleration.

To obtain a relationship between the angular displacement $\theta$ and time $t$, then for different conditions we obtain the following solutions.

\section{(1) No frictional resistance (ideal)}

Here the viscous frictional term $f(d \theta / d t)$ and the coulomb frictional term $(k)$ both reduce to zero and a $₹$

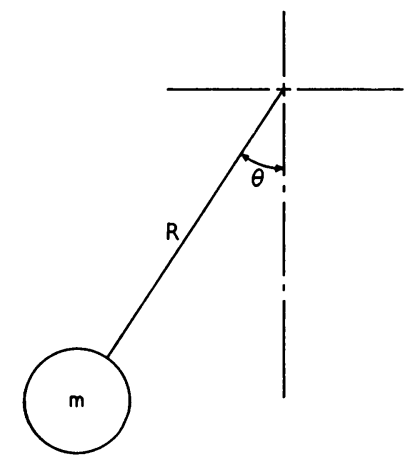

FIG. 1 Diagrammatic representation of a pendulum 
solution of the remaining equation becomes:

$$
\theta=\theta_{1} \cos \omega \mathrm{t},
$$

where $\omega=\sqrt{g / R}$ and $\theta_{1}$ is the initial amplitude, when $t=0$. This of course is simple harmonic motion.

\section{(2) Coulomb frictional resistance at the fulcrum}

In this case the viscous term only equates to zero and the resulting solution for displacement becomes:

$$
\theta=\left(\theta_{1}-Y / \omega^{2}\right) \cos \omega t \pm Y / \omega^{2}
$$

(this must be applied in periods of $\pi$ only), where $Y=k / m R^{2}$.

In this case the amplitude of the motion is seen to decay linearly with time.

\section{(3) Viscous frictional resistance at the fulcrum}

Here the coulomb frictional term equates to zero and the solution for angular displacement becomes:

$$
\theta=\theta_{1} \exp (-\mathrm{at} / 2)\left[\cos \sqrt{\omega^{2}-(\mathrm{a} / 2)^{2}} \mathrm{t}\right]
$$

where $a=f / m R^{2}$.

Hence the amplitude ' $\theta_{n}$ ' reduces exponentially with time.

It can be seen, therefore, that the nature of the resulting relationship between amplitude and time will in principle predict whether a joint possesses the properties of viscous frictional resistance, coulomb (or dry) friction, or no friction at all. Several workers then tested human joints in this manner and predicted the mode of lubrication, as reported above. It is worth pursuing the analysis further, particularly in the case of a fulcrum damped by viscous forces.

Remembering that $a=f / m R^{2}$, with a knowledge of $f$, an order of magnitude for $a$ can be obtained.

\section{Estimation of $\mathrm{f}$}

Assume a hemispherical seat of radius $r$ and a ball separated from each other by a thin film of lubricant of viscosity $\eta$. This film may be assumed to be parallel throughout the head and of very small proportions $h$. Then by calculating the viscous resistance offered by a sliding velocity (angular) of $d \theta / d t$ we can obtain:

$$
f=\frac{4 \pi r^{4} \eta}{3 h}
$$

Therefore

$$
a=\frac{4 \pi \eta r^{4}}{3 h R^{2}}
$$

Consider now the values for the parameters in Equation 2.

$r$, the radius of a normal head, say $20 \mathrm{~mm}$.

$R$, the radius of the pendulum machine from the fulcrum to the mass, $500 \mathrm{~mm}$.

$\eta$, the viscosity of the fluid separating the ball and socket, $0 \cdot 020 \mathrm{Ns} / \mathrm{m}^{2}$. $m$, the mass of the pendulum in these tests, $15-150$ kg.

$h$, the film thickness of the fluid, which cannot be smaller than the surface roughness of the femoral head (assume this value) $2.5 \mu \mathrm{m}$.

When these are substituted into Equation 2, then $a$ is seen to te of the order of $10^{-3}-10^{-4}$. This very small exponent when included in Equation 1 implies that the exponential nature of the relationship is so slight that very sensitive instrumentation would be required for its measurement. Add to this the fact that in a real situation using a natural joint in a pendulum machine, one mechanism is unlikely to act in isolation, any viscous action being expected to remain undetected by systems which measure amplitude of motion of the pendulum.

Recognizing these limitations, a new pendulum was built, but in this model the frictional torque within the femoral head was measured directly using a special sensing head device.

\section{Apparatus}

The apparatus is described in detail by Unsworth, Dowson, and Wright (1975) and is shown in Fig. 2. The acetabular component was held in the measurement carriage by means of plaster which secured it to a ring. This carriage was supported on hydrostatic bearings which meant that very little friction was present in the support carriage (coefficient of friction 0.00001 ) and therefore the measured friction arose purely from the sliding of the joint itself.

The femoral head was fitted into a ring using plaster of Paris and this ring secured in the pendulum itself. The two halves of the joint were then fitted together and loads applied to the bottom of the pendulum. A lever activated a jacking mechanism which lifted the pendulum and separated the two joint surfaces. This allowed the load to be applied suddenly as in walking or jumping while ensuring the same initial angle of swing for each test.

\section{Materials and method}

Six cadaveric hip joints, five normal and one from a patient with severe rheumatoid arthritis, were tested both dry and with their own synovial fluid as lubricant. After dissection of the surrounding tissue the joint was centralized in the fixing rings within the jig. This was then transferred to the pendulum machine in an inverted position. This arrangement allowed any synovial fluid to run into the contact zone.

Tests were carried out at loads in the range 135-1500 N using an initial amplitude of swing of $0.0785 \mathrm{rad}$. The pendulum was held in its initial position $(0.0785 \mathrm{rad})$ by the releasing lever. With the smallest load applied to the pendulum the joint was jacked to disarticulate it. The synovial fluid, previously collected, was admitted to the articular surfaces and the releasing lever was withdrawn. The load was then dropped onto the joint and the oscillations began. The frictional torque experienced by the hip joint was transmitted through the friction measuring 


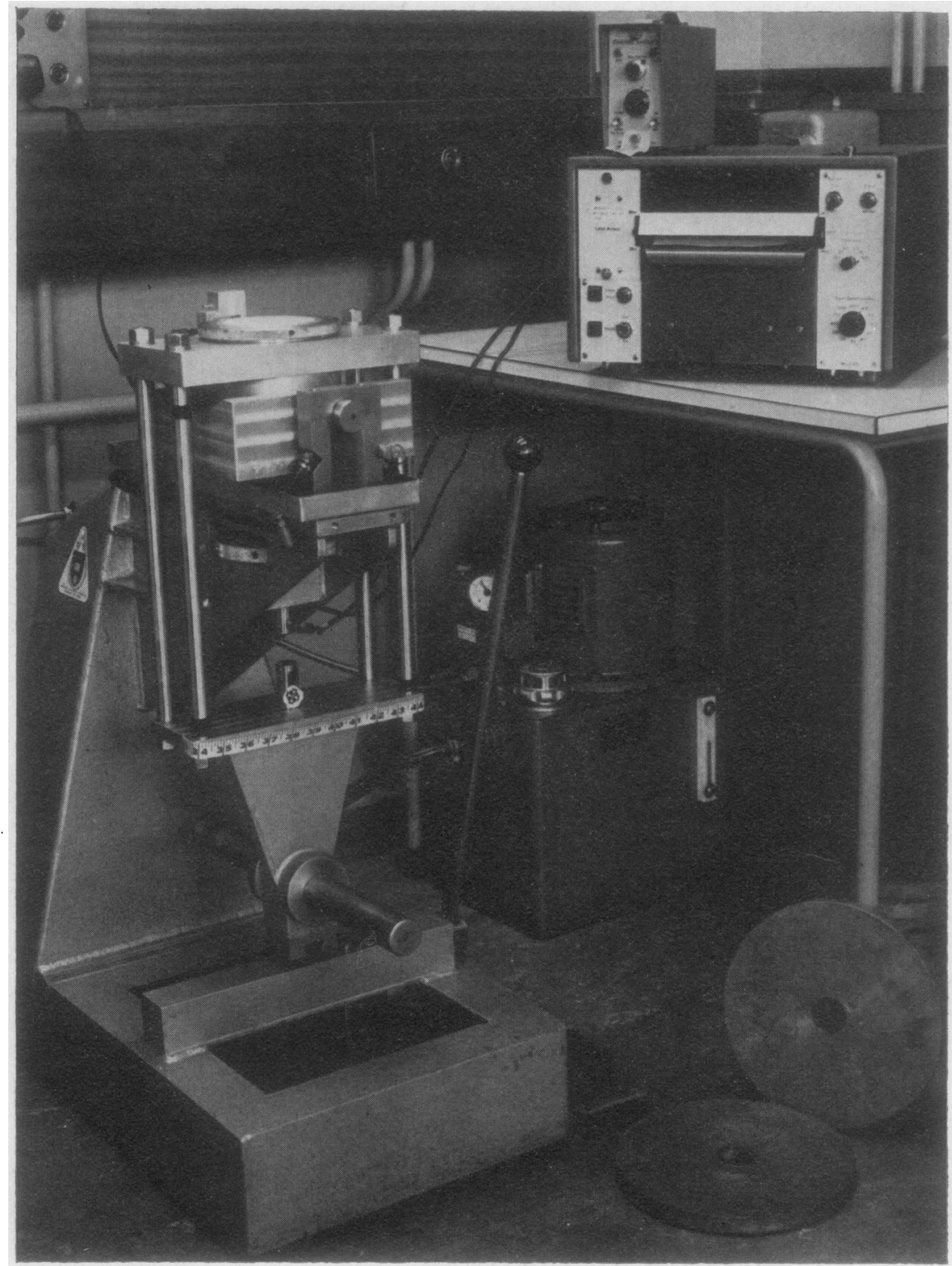

FIG. 2 General layou信 of the pendulum

carriage to the transducer and finally displayed on the trace of an ultraviolet light recorder. In this way frictional torque was measured directly from the instant of loading until the pendulum came to rest. The load was increased in increments of $220 \mathrm{~N}$ carrying out the same procedure at each load. After each test the joint was wiped dry. The experiment was then repeated but the second time applying no lubricant to the joint.

\section{Results}

SUDDENLY APPLIED LOAD TESTS

Fig. 3 shows the results when a load was suddenly applied and the joint was lubricated with synovial fluid. At light loads the coefficient of friction was generally slightly higher than at higher loads, but the interesting point about these results is the shape of the curves. In all cases the coefficient of friction rose with the number of swings of the pendulum, up to a maximum. At this the friction fell again slowly untito finally a sudden fall in the friction marked the end of the tests.

To understand the shape of the curves it is necessary to look at the conditions of loading. At the start of theo test the femoral head is dropped under load onto as film of synovial fluid which is coating the acetabulum. 


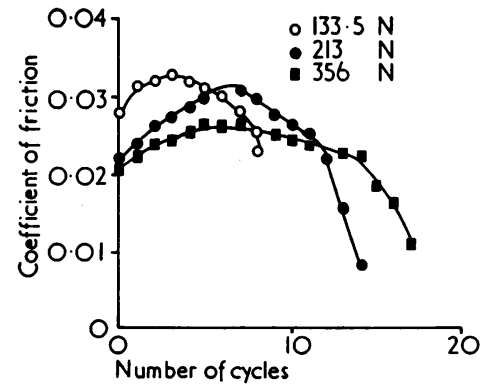

FIG. 3 Natural hip joint No. 2-suddenly applied loads with synovial fluid

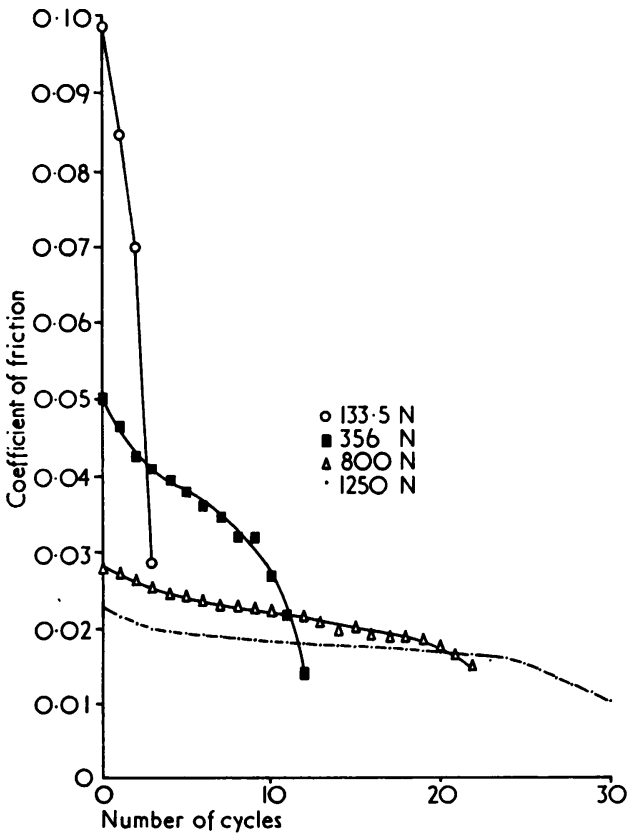

FIG. 4 Natural joint No. 2 dry-suddenly applied loads

The viscous resistance offered to motion is therefore proportional to the sliding velocity but inversely proportional to the fluid film thickness. Thus, as the load sinks through the fluid film the friction will rise producing the observed effect. A point will then be reached where the film thickness is determined by the sliding velocity and the load if an elasto-hydrodynamic process is involved; at this point the friction will reduce as sliding speed reduces, but will increase as the film thickness reduces due to the lower sliding speed. Hence, a roughly constant portion is observed after the increase in friction. The sudden fall in friction marking the end of the test is discussed below.

Fig. 4 shows results from the same joint, but in this experiment all the synovial fluid was wiped away. The shapes of the graphs are now considerably different though the friction still reduced as the load increased. There was no rise in friction initially even though the load was again suddenly applied, supporting the suggestion that this was due to squeeze film action previously. The friction reduced as the number of oscillations increased, which is connected with an interdependence of friction and amplitude of motion.

\section{PRELOADED JOINTS}

If the joint was loaded well before the sliding process began then no squeeze film action would be expected. Fig. 5 shows the results for a joint tested with its own synovial fluid as lubricant but without any

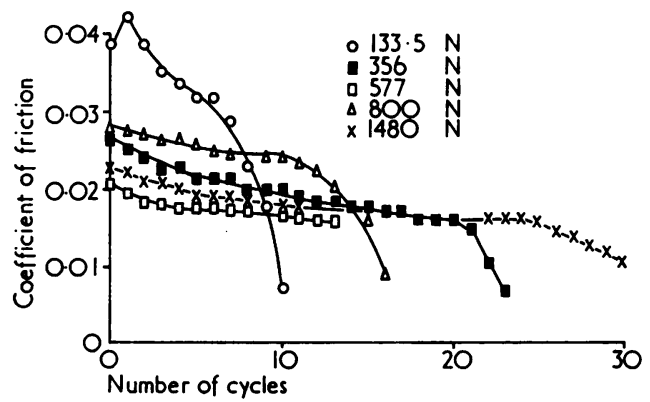

FIG. 5 Natural joint No. 1 lubricated with synovial fluid static loads

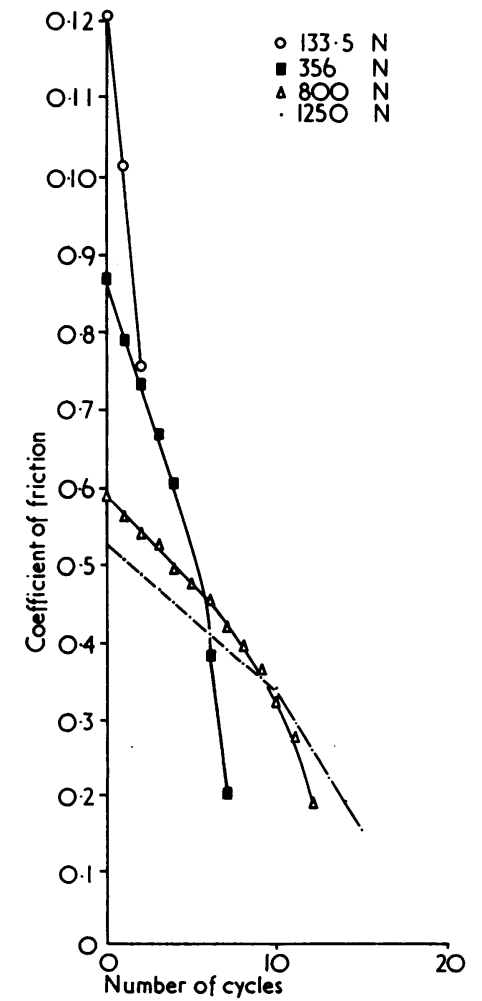

FIG. 6 Natural joint No. 1 dry, static loads 
squeeze film action present. This too supports the view that the rising frictional characteristics shown in Fig. 3 were due to squeeze film action. The same joint when tested dry produced the graph shown in Fig. 6.

\section{Hip joint from patient suffering from rheumatoid arthritis}

The arthritic hip showed a very high coefficient of friction (about 0.36 to 0.41 ). The friction coefficient did not seem to be dependent on the load applied to the joint but it appeared to be amplitude dependent.

\section{Squeeze film lubrication}

Although theoretical studies as long ago as 1886 (Osborne Reynolds) have suggested that squeeze film lubrication is important in animal joint lubrication, previous workers have not shown this. Indeed, some have commented that they did not observe any fluid film action at all (Charnley 1960; Little and others, 1969). In the present studies the presence of squeeze film action seems inescapable. When the joint has a lubricant present and a load is suddenly applied the friction rises with time or number of swings of the pendulum. When the joint is dry but still dynamically loaded, this evidence is not found, as also in the case when the joint is lubricated but a static load is applied. The results therefore obtained during this work support squeeze film action in normal healthy human hip joints.

\section{EFFECTS OF SYNOVIAL FLUID ON THE \\ FRICTIONAL RESISTANCE OF A JOINT}

When synovial fluid is present in a joint the friction is lower than when it is absent. Fig. 7 shows the maximum values of friction recorded in Figs 3 and 4 plotted against load.

In the dry joint the coefficient of friction reduces as the load increases. If pure boundary lubrication were present in such joints a constant coefficient of friction would be expected for all loads. It seems likely, however, that the boundary conditions were changing as the tests progressed, due to the fact that cartilage contains a large quantity of fluid within its physical structure (Edwards, 1966-67), and as a higher load is applied to the cartilage surface the amount of fluid expelled from the cartilage increases. This fluid is then free to be drawn into the contact areas as the joint oscillates, providing some sort of mixed lubrication regimen.

After conclusion of the tests the joint surfaces were wet, supporting the view that a lubricant was present at the end of the tests under high loads in spite of the fact that the joint was dried before the tests began.

The lubricated tests carried out under suddenly applied loading conditions showed that the coefficient of friction was considerably lower at low loads than

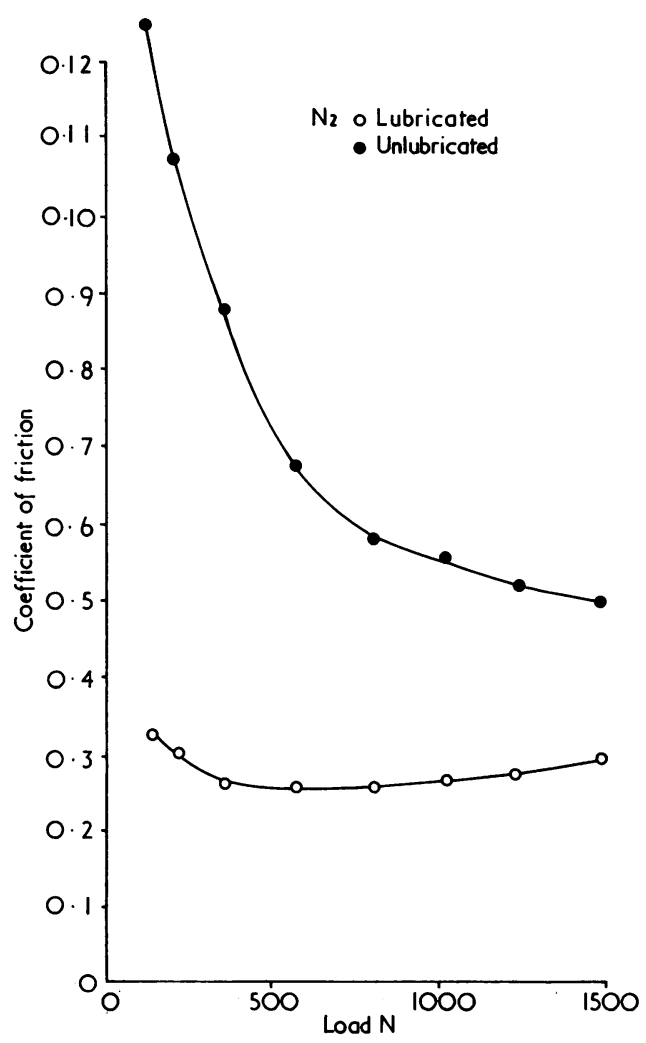

FIG. 7 Suddenly applied loads on a natural joint

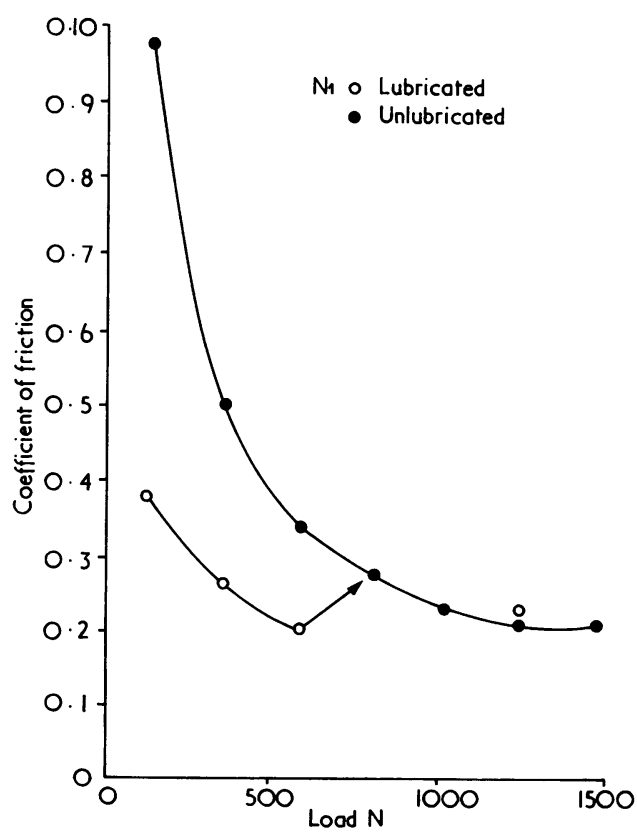

FIG. 8 Static loads on a natural joint 
in tests on dry joint. At higher loads this difference was much less, but it is still advantageous to use synovial fluid.

In the statically loaded hip joint (Fig. 8), at low loads frictional values were considerably lower when a lubricant was applied than when the joints were tested dry. However, at a load of $890 \mathrm{~N}$ there was a sudden change in the frictional value of the lubricated joint such that it corresponded to the value obtained from the dry joint. At all higher loads the two sets of results from the lubricated and unlubricated tests are the same. It can therefore be concluded that synovial fluid only gives an advantage at low loads (less than body weight) unless these happened to be dynamic in nature and hence incorporating the powerful squeeze film mechanism.

\section{PRESENCE OF SELF-GENERATED FLUID FILMS}

The difference between Figs 7 and 8 is important when trying to decide the relevance of self-generated fluid films such as hydrodynamic or elastohydrodynamic lubrication processes. Despite the fact that earlier workers using pendulum machines did not find evidence of any fluid films, elastohydrodynamic or squeeze films, the present studies provide strong evidence of their existence.

In considering Fig. 7, there are several advantages of the lubricated joint over the dry joint. Squeeze film lubrication is certainly one factor but any elastohydrodynamic action could be masked by the squeeze film evidence. However, in Fig. 8 no squeeze film action could be present since steady conditions of loading prevailed. However, a lower coefficient of friction was still observed at low loads when the joint had a lubricant present. This suggests that the synovial fluid was forming a film separating the cartilage surfaces. The unlubricated joint was unable to do this because insufficient fluid could be expelled from the cartilage at low loads to provide lubrication. At higher loads, though, it becomes evident that in the absence of squeeze film action the two tests (initially dry and initially lubricated) gave the same results. This suggests that under higher loads the cartilage expels sufficient fluid to be able to provide a thin film sufficient to carry a load comparable with a fully lubricated joint.

\section{EFFECTS OF TIME UNDER LOAD}

Previous workers (McCutchen, 1959; Walker and others, 1968) noticed that the frictional resistance of cartilage when lubricated increased with the length of time under load. McCutchen explained this in terms of wringing out of the liquid from the cartilage structure, and this explanation seems likely since as this wringing process occurs the area of contact will increase due to the creep of the cartilage and the frictional force will consequently increase. During our normal tests, lasting only about one minute, no evidence of increased friction with time was seen. When, however, a joint was loaded with $1490 \mathrm{~N}$ and tested at various intervals of time after first applying the load, the results show an increasing frictional resistance with time under load. This phenomenon therefore agreed with our previous work (Walker and others, 1968) and highlights the problems of taking measurements of friction from materials of such rheological complexity.

\section{REDUCTION IN FRICTION TOWARDS THE END} OF A TEST

If the friction is increasing as the synovial fluid film reduces due to squeeze film action and a reduction in sliding velocity, it might be expected that when the film breaks down completely the friction will rise to terminate the test. However, in the experiments on natural human joints this did not occur. A possible explanation for the opposite behaviour observed is that the friction force at some point in the test equals the force developed by the shear deflection of the cartilage. As the pendulum thus reduces in amplitude the apparent frictional resistance also reduces to zero as the pendulum comes to rest. In other words, we are not measuring the sliding friction force once we reach a certain point but are recording cartilage resistance to shear forces imposed. Indirect evidence in support of this is provided by tests on a metal-onplastic ball and socket joint where the plastic has a much higher shear modulus than cartilage. In this case the expected rise in frictional force takes place when the joint comes to rest.

\section{EFFECTS OF RHEUMATOID ARTHRITIS}

It is known that stiffness of the joints is a feature of rheumatoid arthritis, and Wright and Johns (1960) described how the stiffness of the metacarpophalangeal joint increases with the disease. In their experiments Wright and Johns found that the greatest contribution to the measured stiffness was from the periarticular structures and that the articular surfaces did not contribute greatly to the overall stiffness. However, in this work the joint surfaces were not loaded in any way and therefore the contribution of the surface friction would be much lower than that for a load-bearing joint such as the hip.

The experiment reported here in which a severely damaged rheumatoid hip was used as fulcrum of a pendulum and tested under a range of loads up to $1500 \mathrm{~N}$, shows that the coefficient of friction reached a value of about 0.4 . This is considerably greater than normal hip joints (0.025) and represents a large increase in joint stiffness as evidenced by the patient. In this particular case the effects of the surface friction would make a significant contribution to the total stiffness experienced. 


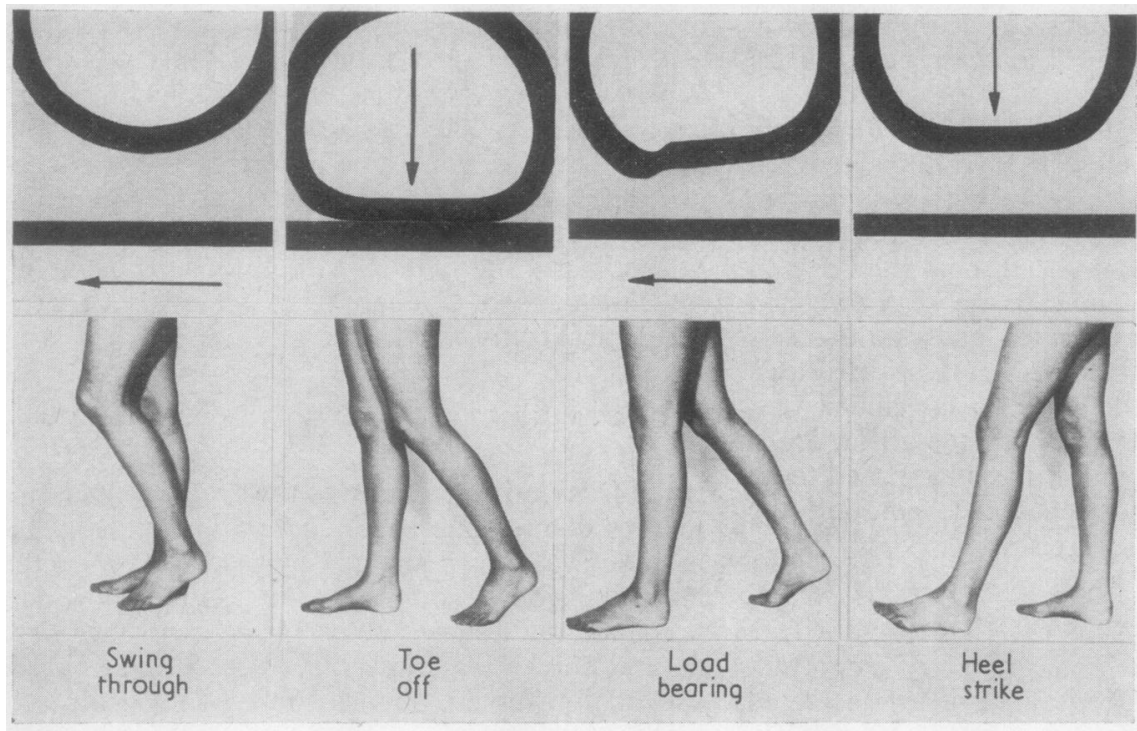

FIG. 9 Lubrication mechanisms in a walking cycle

\section{Conclusions}

Pendulum machines using human joints as fulcra have been examined and the analysis shows that these can be useful, provided the frictional torque is measured directly. Amplitude against time data can be misleading when trying to interpret lubrication regimens.

The results obtained from such a pendulum indicate that human joints make use of squeeze film action and some form of elastohydrodynamic lubrication as well as boundary lubrication. In the walking cycle (Fig. 9), at heel strike there is a load of about three times body weight acting on the hip but there is no sliding motion, since the leg is at its extreme forward position (Paul, 1966-67). At this point the only possible lubrication mechanism is squeeze film. As walking continues, a lighter load is produced and a sliding motion occurs which will draw fluid into the load-bearing area of the joint. At these lower loadsco and higher sliding speeds experiments show that $a_{\bullet}$ fluid film can be generated, thus maintaining t $\mathrm{Be}-\mathrm{J}$ surface separation. When the leg reaches its extrefé posterior position the load is over four times bo $\overline{\text { fyc }}$ weight due to the muscle action in propelling the body forward. At this point again squeeze filmo action will reduce the film thickness and it is possible that under these conditions, when the joint starts $\mathrm{D}$ to move again, the principal mode of lubrication $\overrightarrow{\vec{O}}$ will be boundary. However, this load quickly falls 3 to a low value and the sliding speed increases, producing a regenerated film thickness to protect the surfaces through the cycle once more.

We would like to thank Mr. B. Jobbins and Mr. D. Darby for assistance in the design and commissioning of the apparatus used in this work, and the Arthritis and Rheumatism Council for providing financial support.

\section{References}

Barnett, C. H., AND CobBold, A. F. (1962) J. Bone Jt. Surg. 44B, 662 (Lubrication within living joints)

Charnley, J. (1959) 'Lubrication of animal joints', in 'Biomechanics, Proceedings of the Symposium, 1959', p. 12. Institution of Mechanical Engineers, London.

- (1960) Ann. rheum. Dis., 19, 10 (The lubrication of animal joints in relation to surgical reconstruction by arthroplasty)

Dintenfass, L. (1963) J. Bone Jt. Surg., 45A, 1241 (Lubrication in synovial joints: a theoretical analysis. A rheological approach to the problems of joint movements and joint lubrication)

Dowson, D. (1966-67). Paper 12. Proc. Instn. Mech. engrs., 191 (3-J), 45 (Modes of lubrication in human joints)

EDwards, J. (1966-67) Paper 6. Proc. Instn. Mech. engrs., 181 (3-J), 16 (Physical characteristics of articular cartilage) JONES, E. S. (1936) Lancet, 1, 1043 (Joint lubrication)

LINN, F. C. (1968) J. Biochem., 1, 193 (Lubrication of animal joints: II. The mechanism)

- (1969) Trans. Amer. Soc. Mech. Eng., 91, Ser. L. No. 2, 329 (Lubrication of animal joints)

- AND RADIN, E. L. (1968) Arthr. and Rheum. 11, 674 (Lubrication of animal joints: III. The effect of certain chemical alterations of the cartilage and lubricant) 
Little, T., Freeman, M., AND Swanson, A. (1969) 'Experiments on friction in the human hip joint', in 'Lubrication and Wear in Joints', ed. V. Wright, p. 110. Sector, London

Maroudas, A. (1966-67) Proc. Instn. Mech. engrs., 181 (3-J), 122. (Hyaluronic acid films)

MACConAILl, M. A. (1932) J. Anat. (Lond.), 66, 210 (The function of intra-articular fibrocartilages with special reference to the knee and inferior radio-ulnar joints)

MCCUTCHEN, C. W. (1959) Nature, 184, 1284 (Sponge-hydrostatic and weeping bearings)

- (1966) Fed Proc., 25, 1061 (Boundary lubrication by synovial fluid: demonstration and possible osmotic explanation)

Paul, J. P. (1966-67) Proc. Instn. Mech engrs., 181 (3-J), 8 (Forces transmitted by joints in the human body)

Radin, E. L., SwanN, D. A., AND Weisser, P. A. (1970) Nature, 28, 377 (Separation of hyaluronate-free lubricating fraction from synovial fluid)

ReYNolds, O. (1886) Phil. Trans. Roy. Soc. London, 177, (Part 1), 157 (On the theory of lubrication and its application to Mr. Beauchamp Towers experiments, including an experimental determination of the viscosity of olive oil)

TANNER, R. I. (1966) Phys. med. Biol. 11, 119 (An alternative mechanism for the lubrication of synovial joints)

Unsworth, A., Dowson, D., AND Wright, V. (1975) Trans. Amer. Soc. Mech. Eng. (in press) (The frictional behavior of human synovial joints-Part 1, Natural joints)

Walker, P. S., Dowson, D., Longfield, M. D., AND Wright, V. (1968) Ann. rheum. Dis., 27, 512 (Boosted lubrication in synovial joints by fluid entrapment and enrichment)

Wright, V., AND JoHns, R. J. (1960) Arthr. and Rheum., 3, 328 (Observations on the measurement of joint stiffness) 